\title{
Erratum to: Women Make Movies on the Web: Digital Platforms as Alternative Circuits
}

\author{
Rosanna Maule
}

Erratum to:

Chapter 2 in: R. Maule, Digital Platforms and Feminist Film Discourse, DOI 10.1007/978-3-319-48042-8_2

The Publisher regrets that in the original version of Chapter 2 Fig. 2.2 was published incorrectly. Therefore, we have removed former Fig 2.2 entirely along with its citation.

The updated online version for this chapter can be found at DOI https://link.springer.com/chapter/10.1007/978-3-319-48042-8_2 